\title{
High Resolution DOA Estimation for Vehicular Radar and Communication Integration System
}

\author{
Juan $\mathrm{MA}^{1, \text { a }}$, Xiaoyou $\mathrm{YU}^{1, \mathrm{~b}^{*}}$, Heng $\mathrm{YE}^{1}$, Yan $\mathrm{ZHOU}^{1}$, Nengda $\mathrm{Cl}^{1}$, Pengshuai \\ SUN $^{1}$, Zhinian LUO ${ }^{1}$ \\ ${ }^{1}$ Department of Communication Engineering, School of Information Science and Engineering, \\ Hunan University, Changsha, 410082, China \\ aemail: 295679756 @qq.com, bemail: yu.xiaoyou@163.com
}

Keywords: DOA; Vehicle-to-Vehicle (V2V) Communication; Digital Beam Forming

\begin{abstract}
A new DOA estimation method with high resolution is proposed in this paper for vehicular radar and communication integration system in V2V scenario. This method uses digital beam forming algorithm to estimate the weight vector, from which the DOA of other vehicles can be estimated. Firstly, we presents a new digital beam forming algorithm. Secondly, we can estimate the weight vector based on the new algorithm, and then form the direction map. Finally, the DOA can be obtained by determining the maximum value of the direction map. Through simulation and analysis, the proposed method can obtain higher resolution.
\end{abstract}

\section{Introduction}

Internet of things (IOT)[1]is a new technology, which has been widely used in the existing industrial system. Intelligent transportation systems (ITS)[2]play a very important role in the IOT. Internet of vehicle[3](IOV) is the application of IOT in the ITS. The research of radar communication integration system is important to the IOV. The vehicle radar communication integration system[4]needs some advanced intelligent technology to make it sense actively the changes of the environment. Smart antenna[5]can obtain a larger channel capacity without increasing the band-width. The core technology of smart antenna is the digital beam forming algorithm. And this paper combines digital beam forming with OFDM system[6], which can improve radar sensing ability.

DOA estimation is the important direction to achieving vehicle radar communication integration system. CBF[7]is first proposed as a method, which can estimate the DOA searching the maximum power. Burg proposed the maximum entropy algorithm by using autocorrelation function[8], which largely improve the precision of DOA estimation. Capon[9] proposed a spectrum estimation algorithm - Capon algorithm. MUSIC algorithm[10] was proposed by Schmidt, which utilizes orthogonality between signal and noise subspace. According to the orthogonality of subspace, DOA can be effectively estimated, but its resolution need to be improved.

In view of the above problems, this paper proposes a DOA estimation algorithm based on digital beam forming. We can search the peak value of the direction map to estimate the DOA. Simulation results show that the proposed method has higher resolution.

\section{The application scenario model}

Figure 1 shows the application scenario model for vehicular radar in the V2V environment, where the dotted line represents that each vehicle in the road not only send signals, but also receive signals from other vehicles. Digital beam forming technology can suppress the interference signal and improve the signal to noise ratio. We can extract useful information to estimate the DOA more accurately by the relevant algorithm. 


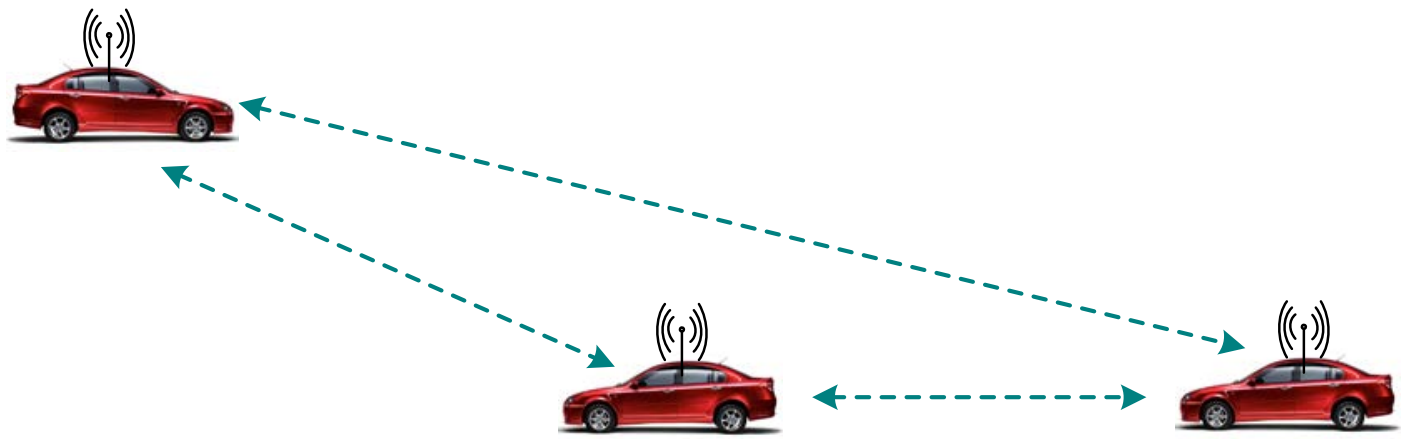

Figure.1 The application scenario model

\section{Signal model}

After the IFFT transformation, the frequency domain signal of each antenna becomes the time domain signal, its expression is as follows:

$$
s_{l, \mathrm{q}}(\mathrm{t})=\sum_{\mu=0}^{N_{\text {sym }}-1} \sum_{c=0}^{N_{c}-1} y_{l}\left(\mu N_{c}+\mathrm{c}\right) \cdot \exp \left(\mathrm{j} 2 \pi\left(\mathrm{q}+\mathrm{c} \cdot N_{T X}\right) \Delta f t\right)
$$

Where $S_{l, q}(\mathrm{t})$ is the signal of thel-th user by the $(q+1)$-th antenna. $y_{l}$ is the modulated symbol of the $l$-th user, which includes pilot signal $y_{l, p} . N_{s y m}$ denotes the number of OFDM symbols in a frame and $N_{T X}$ represents transmit antenna number. $N$ is the number of subcarriers in an OFDM symbol. $N_{c}$ is the number of sub-carriers in an OFDM symbol on each transmit antenna and $N_{c}=N / N_{T X} . \Delta f$ denotes the subcarrier spacing. According to the orthogonality of complex signals, there is $\Delta f=1 / T$.

The receiver uses the uniform linear array (ULA) to receive the signal consisting the transmission signal of $\mathrm{L}$ vehicle. $\mathrm{M}$ and $d$ denotes array element number and array element spacing. $\theta_{l}$ denotes the DOA for the $l$-th vehicle, which is defined as the included angle between the arrival direction of the $l$-th signal and the array line. Receiving signal expression can be obtained by derivation.

$$
\boldsymbol{X}(t)=\boldsymbol{A S}(t)+\boldsymbol{V}(t)
$$

Where $\boldsymbol{X}(t)=\left[\begin{array}{llll}x_{1}(\mathrm{t}) & x_{2}(t) \quad \ldots \quad x_{M}(t)\end{array}\right]^{T}$ and $\quad x_{m}(t)$ is receiving signal of the $m$-th element, which is expressed as follows:

$$
x_{m}(t)=\sum_{q=0}^{N_{T X}-1} \sum_{l=1}^{L} b_{l, q} \exp \left(-\mathrm{j} 2 \pi(\mathrm{m}-1) \frac{d \sin \theta_{l}}{\lambda}\right) s_{l, q}(t)
$$

Where $\lambda$ is wavelength and $b_{l, q}$ represents attenuation amplitude. In formula (2), $\boldsymbol{A}$ is the steering vector of the antenna array, $\boldsymbol{A}=\left[\begin{array}{llll}\mathbf{a}\left(\theta_{1}\right) & \mathbf{a}\left(\theta_{2}\right) & \ldots & \mathbf{a}\left(\theta_{L}\right)\end{array}\right]$. Where $\mathbf{a}\left(\theta_{l}\right)$ denotes the array steering vector of the $l$-th vehicle, $\mathbf{a}\left(\theta_{l}\right)=\left[1 \exp \left(-\mathrm{j} 2 \pi \mathrm{d} \sin \theta_{l} / \lambda\right) \ldots \exp \left(-\mathrm{j} 2 \pi(\mathrm{M}-1) \mathrm{d} \sin \theta_{l} / \lambda\right)\right]^{T}$. Where $\boldsymbol{S}(\mathbf{t})=\left[\begin{array}{lllll}\mathrm{s}_{1}(t) & \mathrm{s}_{2}(t) & \ldots & \mathrm{s}_{L}(t)\end{array}\right]^{T}$ and $s_{l}(t)=\sum_{q=0}^{N_{T X}-1} b_{l, q} s_{l, q}(t) \cdot \boldsymbol{V}(\mathrm{t})$ is the gauss white noise vector, and $\boldsymbol{V}(\mathbf{t})=\left[\begin{array}{lllll}v_{1}(t) & v_{2}(t) & \ldots & v_{M}(t)\end{array}\right]^{T}$.

\section{DOA estimation}

In the receiver, the received signal is transformed by A/D converter, and the phase information is still retained. After the FFT operation and multi-decomposition for A/D converted signal $\boldsymbol{X}$, the pilot signal $\boldsymbol{Y}_{p}$ can be extracted. And then we can get signal $\boldsymbol{X}_{p}$.In order to use the reference pilot 
signal $y_{l, p}$, the pilot signal $\hat{s}_{l, p}$ is matched and filtered by the digital beam forming. The frequency domain pilot signal $\hat{y}_{l, p}$ can be obtained by FFT transformation. According to the digital beam formation algorithm, using the reference pilot signal and estimated reference pilot signal for updating the error signal so as to realize the updating of the weight vector. So we can estimate the weight vector from the vehicles by repeating the cycle. According to the direction function, we can use the estimated weight vector to form a direction map and search the DOA. According to the maximum value in the direction map we can get the DOA from the vehicle.

We can get the weight vector by those processes. (1)Initialization of the weight vector. $W_{l}=0$. (2)The estimation of the error signal. $e_{l}(n)=s_{l, p}(n)-\boldsymbol{W}_{l}^{H}(\boldsymbol{n}) \boldsymbol{X}_{p}(\boldsymbol{n})$, where ${ }^{s_{l, p}}$ is obtained after IFFT transform from frequency pilot signal $y_{l, p}$.(3)Update iterative step. (4)Update weight vector. $\boldsymbol{W}_{l}(n+1)=\left(I-2 \mu(\mathrm{n}) \mathbf{U}_{s s} \boldsymbol{\Sigma}_{s s} \mathbf{U}_{s s}^{\mathbf{H}}\right) \boldsymbol{W}_{l}(n)+2 \mu(\mathrm{n}) \boldsymbol{X}_{p}(n) s_{l, p}{ }^{*}{ }^{(n)}$, where $\boldsymbol{R}_{X X}=E\left[\boldsymbol{X}_{p} \boldsymbol{X}_{p}^{H}\right]$ and $\mathbf{U}_{s s} \boldsymbol{\Sigma}_{s s} \mathbf{U}_{s s}^{\mathbf{H}}$ is signal subspace of $\boldsymbol{R}_{X X}$. Finally we can get the weight vector $\mathbf{W}_{l}$ by repeating those steps.

The direction function can be expressed as follows:

$P(\theta)=\left|\mathbf{W}_{l}^{H} \mathbf{a}(\theta)\right|$

We can see that the direction map has the maximum value at the DOA, and the gain value is smaller in the other direction. Therefore, we can use digital beam forming algorithm to estimate the weight vector, and use the estimated weight vector to form the direction map and we can get the DOA by determining the maximum value of the direction map.

\section{Simulation experiment and analysis}

In order to study the performance of the proposed method, the CAPON algorithm, MUSIC algorithm and the proposed method in this paper will be compared in the following simulation analysis.
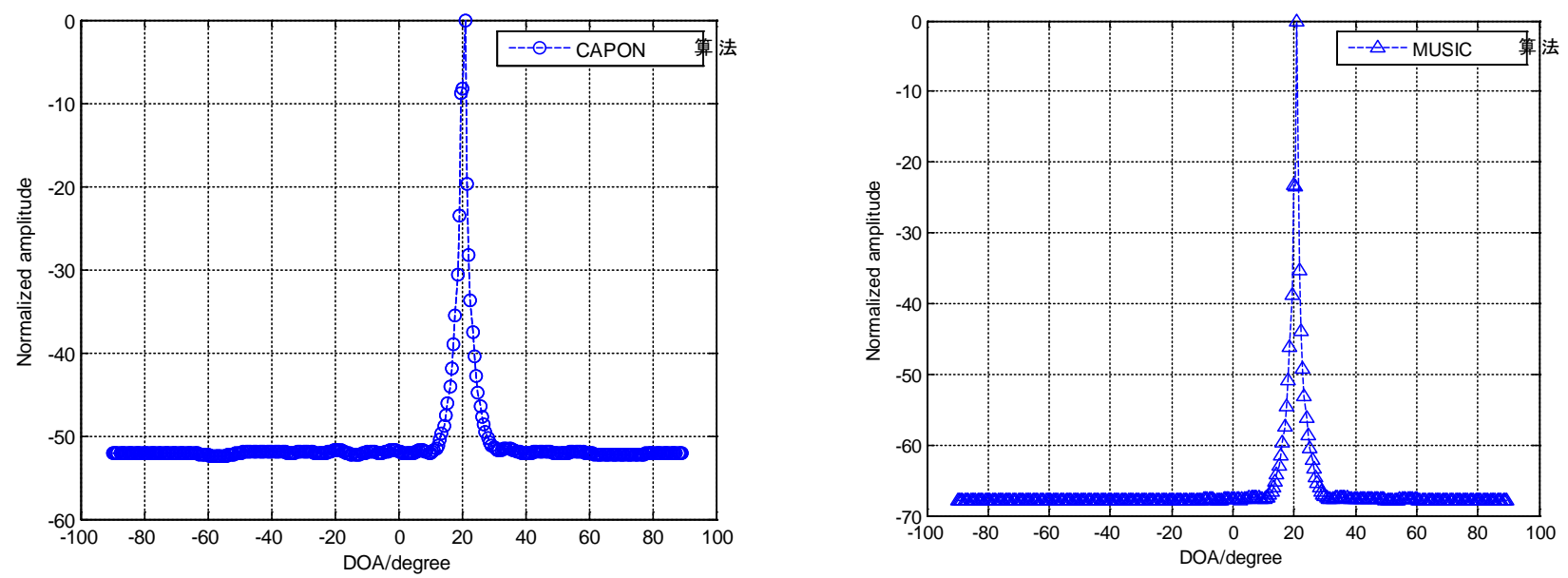

Figure 2 DOA estimation of two algorithms

Assuming the receiving end uses 16 array element to receive the signal .The distance $\mathrm{d}$ between antenna elements is $\lambda / 2$ and the number of snapshots is 200. In this paper, the DOA from the vehicle are $20^{\circ}, 21^{\circ}$. From figure 2, we can see that CAPON algorithm and MUSIC algorithm have only one spectral peak. From figure 3, the proposed method has two peaks, which can estimate DOA successfully. Therefore the resolution of the proposed method is higher than the CAPON algorithm and MUSIC algorithm. 


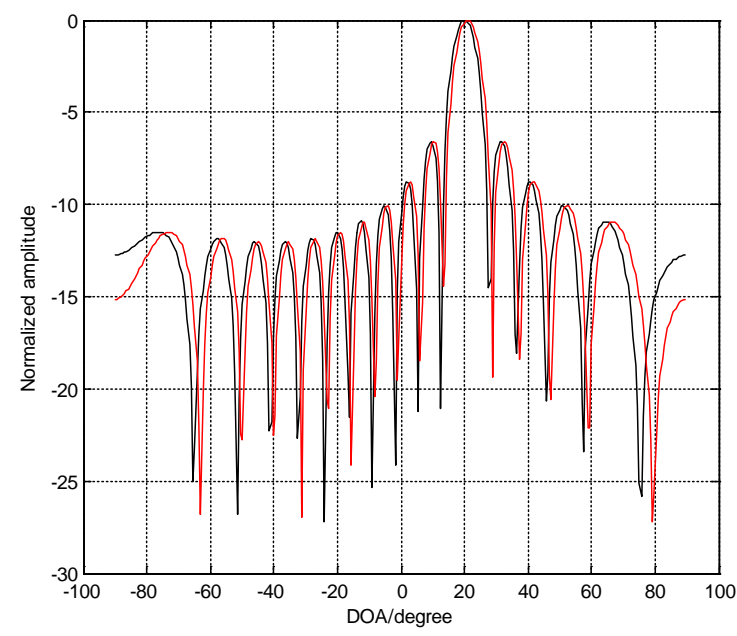

(a) The proposed method

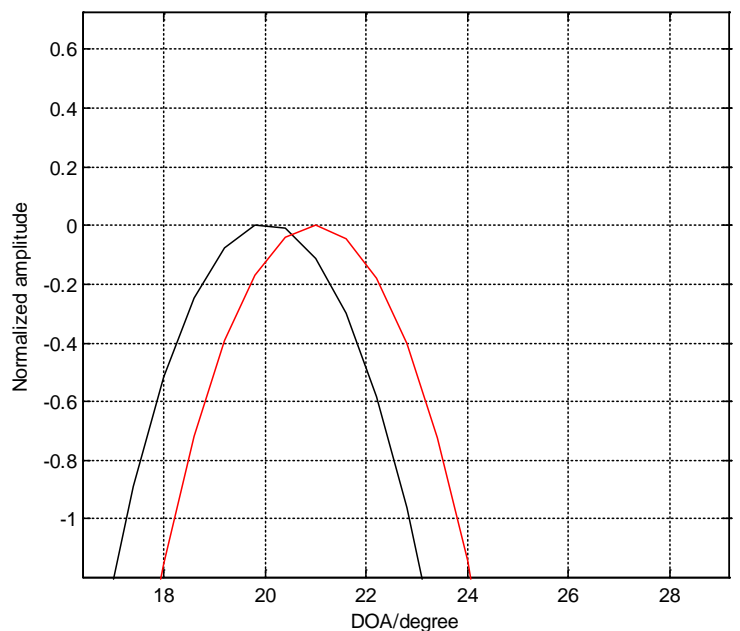

(b) Local magnification of the proposed method

Figure 3 DOA estimation results

\section{Conclusion}

In the vehicle radar communication integration system improvement of resolution in angle estimation, this paper proposes a new DOA estimation method. First, this paper put forward a new algorithm. We can search the peak value of the map to estimate the DOA using the new algorithm to estimate the weight vector to form a direction map. Through the comparative analysis of the CAPON algorithm and MUSIC algorithm, the proposed method has a higher resolution.

\section{Acknowledgement}

In this paper, the research was sponsored by the National Natural Science Foundation of China (Project No. 61371115).

\section{References}

[1] Kolias C, Stavrou A, Voas J, et al. Learning Internet-of-Things Security "Hands-On"[J]. IEEE Security \& Privacy, 2016, 14(1):37-46

[2] Kachroo P, Sastry S. Travel Time Dynamics for Intelligent Transportation Systems: Theory and Applications[J]. Intelligent Transportation Systems IEEE Transactions on, 2016, 17(2):385-394

[3] Ding Z M. A Design of Cooperative Vehicle Infrastructure System Based on Internet of Vehicle Technologies[J]. Applied Mechanics \& Materials, 2014, 552:363-366.

[4] Shen X, Yang R, Li X, et al. The Research on DS-OFDM in Integrated Radar and Communication. Wireless Communications[M]. Networking and Applications. Springer India, 2016

[5] Santos M R, Oliveira B G M D, Melo M T D, et al. A smart antenna system for remote supervision[J]. International Journal of Applied Electromagnetics \& Mechanics, 2014, 45:293-299.

[6] J Mar J, Kuo C C, Tsai F Y. Realization of DBF-OFDM Transceiver for Vehicular Communication Using FPGA Chip[C]. Vehicular Technology Conference, 2012 IEEE 75th. IEEE, 2012:1-5.

[7] Muzic R R, Nelson A D, Miraldi F. Temporal alignment of tissue and arterial data and selection of integration start times for the $\mathrm{H}(2)(15) \mathrm{O}$ autoradiographic CBF model in PET[J]. IEEE Transactions on Medical Imaging, 1993, 12(3):393-8.

[8] Cadzow J A. Maximum Entropy Spectral Analysis[M]. Encyclopedia of Statistical Sciences. 
John Wiley \& Sons, Inc. 1975:1519-1533.

[9]Hirakawa M, Tsuji H, Sano A. Computationally efficient DOA estimation based on linear prediction with Capon method[C]. 2001:3009-3012.

[10] Schmidt R O. Multiple emitter location and signal parameter estimation[J]. IEEE Transactions on Antennas and Propagation, 1986, 34(3): 276-280 